\title{
Cloud Imaging from Meteorological Satellites and its Application to Robotic Observing
}

\author{
Andre Phillips ${ }^{\mathrm{A}, \mathrm{B}}$ and Michael C. B. Ashley ${ }^{\mathrm{A}}$ \\ A Department of Astrophysics and Optics, University of New South Wales, Sydney NSW 2052, Australia \\ B Corresponding author. Email: a.phillips@unsw.edu.au
}

Received 2005 June 24, accepted 2005 September 17

\begin{abstract}
Satellite cloud imagery is a standard tool of meteorology, and also of ground-based observational astronomy. Fast access to cloud imagery through the Internet now permits more accurate prediction of local cloud cover than was formerly the case. This is particularly useful for automatic and remotely operated telescopes, where an observer may be at a considerable distance from the telescope itself. Since satellite imagery can now be accessed through the Internet with such ease, direct reception of radio transmissions from weather satellites may seem unnecessary. However, advances in computing power and radio receiver technology permit simple automated receipt of satellite transmissions, particularly from the NOAA series of low Earth orbit satellites. This has the advantage of more up-to-date, and higher resolution, cloud imagery than can be obtained from the Internet. We have operated such a satellite receiving station since 2003 April, and have found it particularly useful when remotely operating our telescopes at Siding Spring Observatory (SSO). By automatically publishing our cloud imagery onto the World Wide Web we have established a resource that is widely used by other local observatories and by the general public.
\end{abstract}

Keywords: atmospheric effects — site testing — telescopes

\section{Introduction}

Since 1977 the main source of satellite cloud imagery over Australia has been the Geostationary Meteorological Satellite (GMS) series of satellites operated by the Japan Meteorological Agency (JMA). The first GMS satellite was launched in 1977 July and the most recent, GMS-5, launched in early 1995. All GMS satellites were successfully placed into geosynchronous orbit, stationed above longitude $140^{\circ}$ East. Despite a nominal design life of five years, GMS-5 was still in operation during late 2004, at which time it suffered an internal failure and imagery were subsequently acquired ${ }^{1}$ by the US-owned GOES-9 satellite stationed over longitude $155^{\circ}$ East. A replacement for GMS-5, the Japanese MTSAT-1R satellite, was placed into geostationary orbit during 2005 March and during has become the primary source of Australian cloud imagery data. Australia is fortunate that it shares the same geographical longitude as Japan and that satellite cloud imagery is freely shared by the JMA.

From its vantage point $35800 \mathrm{~km}$ above the Earth's equator, a geostationary satellite can observe $42 \%$ of the Earth's surface. These near-hemispherical Earth images, in various spectral bands, are transmitted from GMS-5 at 30-minute intervals, with a typical delay between image acquisition and Internet availability of around one hour. Within Australia, these data are directly received and

\footnotetext{
${ }^{1}$ Images acquired by GOES- 9 were remapped to a GMS-5 projection and then uploaded to GMS-5 for general broadcast.
}

distributed via several sources including the Australian Bureau of Meteorology (BOM) and James Cook University (JCU). These unencrypted satellite broadcasts are also received directly by many interested amateurs, using relatively modest and inexpensive equipment.

In addition to the geosynchronous weather satellites, Australia is routinely overflown by a constellation of low Earth orbit (LEO) satellites operated by the USA's National Oceanographic and Atmospheric Administration (NOAA). Flying in sun-synchronous polar orbits at an altitude of around $800 \mathrm{~km}$, and with an orbital period of around 100 minutes, NOAA satellites continuously transmit radiance data from a strip of terrain oriented orthogonal to the satellite's ground track. By assembling these strips, a near-real-time image of the overflown terrain may be obtained. It is the receipt of this easily received imagery which is the focus of this paper.

\section{UNSW's Robotic Telescopes at Siding Spring}

Weather and moon conditions permitting, we remotely operate the Automated Patrol Telescope ${ }^{2}$ (APT) and UNSW's Robotic Optical Transient Search Experiment (ROTSE; Akerlof et al. 2003) telescopes full time. During 2005, the operation of the APT was mostly conducted from the United Kingdom, where the Australian night time observation schedule corresponded favourably to the normal UK working day. In general our telescopes require

\footnotetext{
${ }^{2}$ www. phys.unsw.edu.au/apt
} 
little intervention during a night's observing, just an occasional check of the meteorological and sky conditions. If the weather deteriorates badly during an evening (e.g. clouds, high humidity, or precipitation) the telescopes will sense the change and automatically shut down. For our present observing programme, which requires precision photometry over large fields of view, the effects of cloud interference are critical.

Cloud cover is monitored from the ground by two allsky cameras that we operate. One camera is a simple low-light-level upward-pointing video camera, and the other is $\mathrm{CONCAM}^{3}$, part of a global array of similar instruments operated by the Michigan Technical University (Shamir \& Nemiroff 2005). The video camera gives good daylight and twilight sky imagery, whereas CONCAM is specialized for a dark sky. Since CONCAM data are continuously assembled into on-line time-lapse animations of the local sky, it is easy to spot the presence of cloud - somewhat more effective in fact, than fully dark-adapted human eyes. But perhaps the most sensitive detector of cloud is our APT telescope itself, which performs real-time photometric analysis of acquired images. Even with this sensitivity to cloud, some types of very thin cirrus cloud are still difficult to detect in the data, and the real-time whereabouts of such cloud is of interest to us. Fortunately the great height of such cloud means it is also very cold, and therefore it is often prominently visible in satellite thermal infrared imagery.

The GMS-5 images of Australia, which are distributed by the BOM and JCU, have a spatial resolution of around $10 \mathrm{~km}$ and a delay of around one hour between image acquisition and web availability. With our desire to monitor local cloud, particularly high thin cirrus, we have experimented with the direct real time reception of NOAA LEO satellites. Although NOAA satellite overflights occur only about five to seven times a night, their image resolution is quite good at around $4 \mathrm{~km}$, and we can place the data on the Web in near real-time. We have found this immediacy and resolution particularly useful and, judging from the frequent access to our website ${ }^{4}$, so have many others.

\section{NOAA Satellites}

The NOAA Polar Earth Orbiting (POES) series of satellites are a joint development between NASA and NOAA, and they have been in operation since 1978. All NOAAPOES spacecraft have been placed in similar sun synchronous $^{5}$ near-circular polar orbits. With a mass of approximately 1.4 tonnes and physical dimensions comparable to that of an automobile, they are large sophisticated satellites carrying a large array of sensors. Of particular interest to astronomers is the Advanced Very High Resolution Radiometer (AVHRR) instrument, which scans a strip of the Earth orthogonal to the orbital ground

\footnotetext{
${ }^{3}$ http: / / nightskylive. net

${ }^{4}$ Our website receives typically 800 hits per day of which approximately $40 \%$ are from within Australia.

${ }^{5}$ Orbital inclination $98.7^{\circ}$, period 101 minutes, altitude $800 \mathrm{~km}$.
}

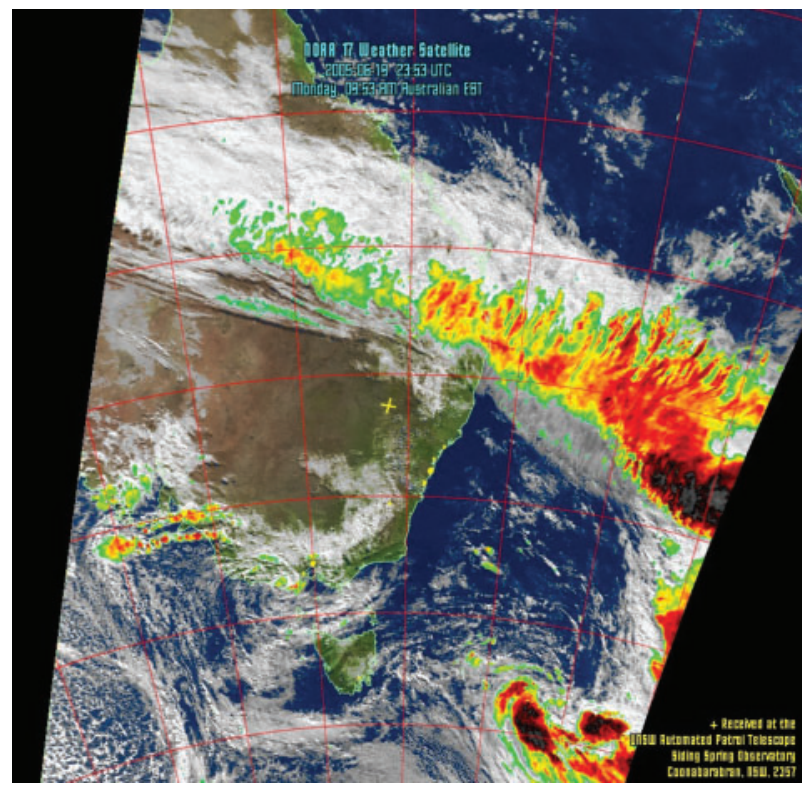

Figure 1 Example of a day-time NOAA APT image, showing temperature colour-coding of high cloud. Cloud is normally coloured white, except when the cloud-top temperature is $<-20^{\circ} \mathrm{C}$, in which case it is coloured green. Such coloured cloud is normally associated with rain, and approaching rain-bearing fronts. Yellow clouds have cloud-top temperatures $<-30^{\circ} \mathrm{C}$ and red $<-40^{\circ} \mathrm{C}$.

track, with a $1.1 \mathrm{~km}$ resolution. The gaze of this instrument is directed towards the nadir of the spacecraft and it scans a strip of Earth $55.4^{\circ}$ either side of the ground track, acquiring radiance data in one visible and five infrared channels. Radiance data are then transmitted in real time in two broadcast formats: High Resolution Picture Transmission (HRPT) and Automatic Picture Transmission (APT). HRPT is a digital format transmitted at a frequency of around $1.7 \mathrm{GHz}$, and which includes information from all six AVHRR channels, and with a spatial resolution of around $1 \mathrm{~km}$. APT, a lower spatial resolution ana$\log$ transmission format incorporating data from just two AVHRR channels, is simultaneously broadcast at a frequency of $137 \mathrm{MHz}$. Despite the lower spatial resolution of approximately four kilometres, NOAA APT still compares favourably with the freely available GMS-5 spatial resolution of about $10 \mathrm{~km}$.

An example of a typical daytime NOAA APT image is shown in Figure 1, which is a composite of visible and thermal-infrared pictures. From the visible light channel, one can observe large scale vegetation features on the ground, and from the thermal-infrared one can measure cloud-top temperature.

During the night, the NOAA APT transmissions change to mid- and thermal-IR channels, an example of which is shown in Figure 2. As is the case with daylight satellite passes, our data reduction program artificially colours land and sea to aid the interpretation of the image.

The radio spectrum between 137.0 and $138.0 \mathrm{MHz}$ is an internationally designated band for satellite communications, and many communications and weather satellites transmit their data here. NOAA APT radio transmissions 




Figure 2 Example of a night-time NOAA APT image, showing temperature colour-coding of high cloud.

are frequency modulated (FM), rather similar in format to regular broadcast FM radio transmissions. When listening to a NOAA APT signal on a FM radio receiver, one hears a prominent $2400 \mathrm{~Hz}$ tone, which is pulsed every half second. This tone is the audio 'subcarrier' which encodes the radiance data (where variation in the ground radiance measurement causes the amplitude of the tone to correspondingly vary). The half-second pulsing of the tone corresponds to the line scan rate, where each half-second pulse corresponds to a single line on the raw NOAA APT image. From a radio receiver, the $2400 \mathrm{~Hz}$ audio tone may be fed into a standard PC 'sound card', where it is digitized. Because of the similarity between the NOAA APT format and standard broadcast-FM radio transmission, equipment is readily available from consumer electronics suppliers and is relatively cheap.

\subsection{The Ground Station Design}

With the computing power of modern PCs and the availability of economical very high frequency (VHF) radio receivers, automatic reception of NOAA APT transmissions is well within the scope of any astronomical observatory, or enterprising high school student. The basic components required are a computer, receiver, antenna, antenna preamplifier, and suitable software to run it all. Each component will now be considered.

\subsection{The Receiver}

So-called VHF 'scanner' radios are generally suitable for receiving $137 \mathrm{MHz}$ FM signals, although the limited passband of the receiver (typically $10 \mathrm{kHz}$ ), may limit the full range of radiance data that may be received. Some better quality receivers have a variable passband, known more typically as the Intermediate Frequency (IF) bandwidth. A receiver IF bandwidth of $20 \mathrm{kHz}$ or more is desirable, but not essential. Many scanning receivers now have a computer control facility, typically through an RS-232 serial connection. This facility is useful for fully automatic reception of NOAA APT signals, to tune the receiver to the specific frequency of a particular NOAA spacecraft transmitter.

\subsection{The Computer}

Any Pentium-class PC, running any popular operating system, with disk storage capacity of a few gigabytes and a random access memory of a few tens of megabytes, is suitable. The computing effort to receive the NOAA APT signal, and to process the image, is modest, so almost any computer is suitable for the task. These modest requirements also mean that the computing task of running a receiving station may simply become a sub-process running on an existing computer that is generally being used for other purposes. This is how we run our satellite receiver station.

\subsection{The Software}

Many good public domain programs for controlling and operating NOAA APT receiver stations exist. However, we have had excellent success with a computer program known as WXтоImg (Anderson 2005). This program, which is written for all common computer operating systems, simultaneously controls the radio receiver, processes the received image, and automatically uploads fresh images to the Web. For us this has meant automatic and trouble-free operation, often for months at a time.

\subsection{The Antenna}

Unlike the high gain antennas and parabolic dishes normally associated with satellite communications, $137 \mathrm{MHz}$ NOAA APT reception normally utilizes simple low-gain omnidirectional antennas. With the minor complication that the NOAA APT radio signal transmitted from the spacecraft is circularly polarized, the antenna technology and operating frequency is similar to normal terrestrial VHF television antennas. This means that standard VHF TV antenna preamplifiers and coaxial feeder cables may be used. Popular designs for polarized omnidirectional antennas include Lindenblad and Quadrafilar Helix antennas (Davidoff 1990; Straw 2003). Much information may be found on the Web concerning the relative merits of these antennas, but in fact extremely simple antennas can still produce good results. More details concerning receiver selection and antenna designs many be found on our web page (Phillips 2005).

\section{The Future of the NOAA APT Format}

The simple analog transmission format of NOAA APT has one big drawback: its susceptibility to ground-based radio interference. The advent of broadcast television, mobile pagers, and consumer electronic devices controlled by microprocessors (such as microwave ovens, VCRs, or dishwashers) has given rise to an enormous increase in urban radio noise at VHF frequencies. In fact, in many 


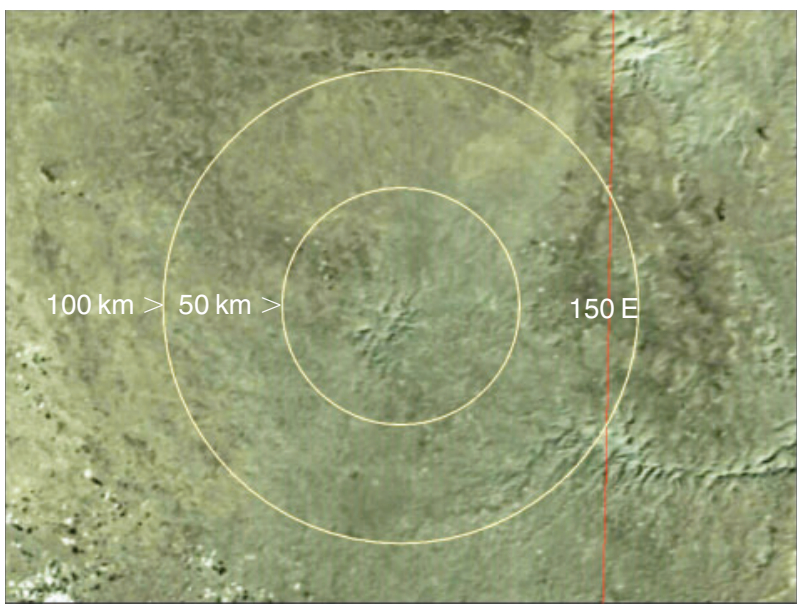

Figure 3 Example of a daytime HRPT image, centred on Siding Spring Observatory, showing local vegetation and geographical features. Yellow rings indicate 50 and $100 \mathrm{~km}$ ranges from SSO, and the vertical red line indicates longitude $150^{\circ}$ East.

city locations, particularly those with a TV tower transmitting Australian channel 5A, NOAA APT reception may be impossible (e.g., within the city of Newcastle, north of Sydney). In due course analog NOAA APT transmissions will be phased out and replaced with a comparable digital format. However, it is likely that NOAA APT will be around for another decade or so. The latest addition to the constellation of NOAA POES satellites was launched on 2005 May 20 and is transmitting regular NOAA APT at 137.9125 MHz. Another identical satellite (NOAA-N') is planned for launch in 2008, and will also transmit regular analog NOAA APT.

The launch of NOAA-N' will mark the end of this series of NOAA spacecraft. Since 1971 the USA Department of Defence has operated a series of polar orbiting meteorological satellites known as the Defence Meteorological Satellite Program (DMSP). For reasons of economy it was decided to merge the NOAA and DMSP meteorological satellite programs, and the successor spacecraft to NOAA-N' ${ }^{\prime}$ will be known as the National Polar-orbiting Operational Environmental Satellite System (NPOESS). NPOESS spacecraft will no longer transmit analog NOAA APT, but instead will transmit an equivalent real-time digital format known as Low Rate Picture Transmission (LRPT) within the $137 \mathrm{MHz}$ space band.

In due course we plan to acquire LRPT or HRPT equipment. At the time of writing a private HRPT station (located in Sydney) has begun operation, and it automatically publishes near-real-time data directly to the Web. An example of a HRPT image of the SSO local area acquired during daylight is shown in Figure 3. During periods of low sun angle, ground topography can be easily observed, particularly the Warrumbungle Ranges (where SSO is located) and Mt Kaputar located $140 \mathrm{~km}$ towards the northwest. Real time image data can be accessed at the HRPT web page (Indermuehle 2005). An example of night-time HRPT imagery of the SSO area is shown in Figure 4. Construction of an HRPT receiving station is

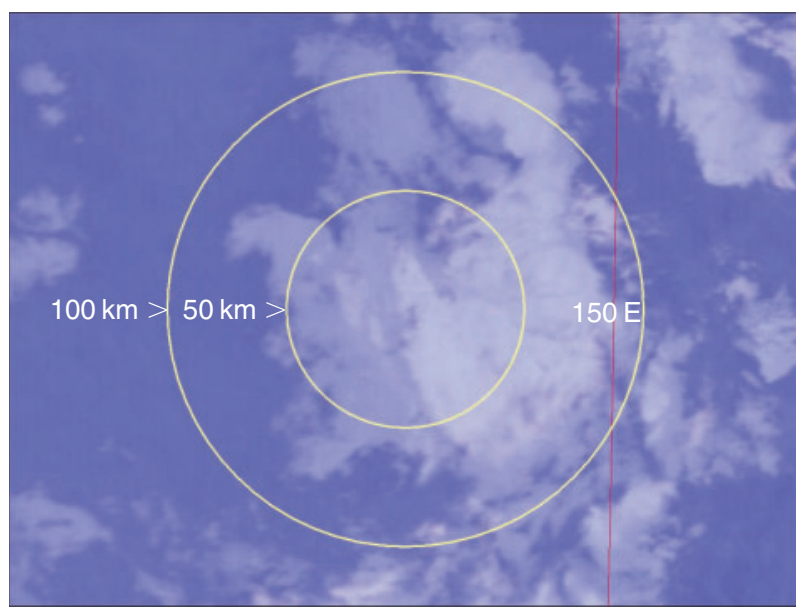

Figure 4 Example of a night-time HRPT cloud image, centred on Siding Spring Observatory. Yellow rings indicate 50 and $100 \mathrm{~km}$ ranges from SSO.

considerably more technically challenging and expensive than for a NOAA APT station, but, as the advent of the new Sydney stations shows, still within the scope of a motivated individual.

\section{Educational Outreach}

Our satellite weather website receives around 1000 hits and 150 visits $^{6}$ per day, many of which come from SSO users. But there is also strong public interest in the site, particularly the web pages that describe home construction of a receiving station. Judging by the number of email queries that we receive, this published information has assisted in the construction of several new NOAA APT receiving stations within Australia. The relative simplicity of directly receiving NOAA APT signals lends itself to education, where an enterprising high school student would have little difficulty assembling the necessary equipment. Brigidine School (located in Randwick, Sydney) is renowned for incorporating satellite communication into its science curriculum, and during 2005 June they even undertook live voice communications with astronauts aboard the International Space Station (Ryan 2005). Our website incorporates several pages of technical notes on how to build a NOAA APT receiving station, and a specialized page directed towards schoolteachers, with relevant educational links. Even if our weather pages are simply viewed by web-surfing school children, we believe that we are providing cost-effective science outreach.

\section{Conclusions}

We have found that the direct reception of NOAA APT satellite imagery is particularly useful for the remote operation of our telescopes. The 4-km spatial resolution of NOAA APT is generally somewhat better than comparable geostationary satellite data. Web availability of cloud imagery occurs within a few minutes of a NOAA

${ }^{6}$ Website visitors may reload pages several times during a single visit, and hence the number of hits exceeds visits. 
satellite pass, and this speed is useful to correlate with real-time cloud motion observed with ground-based allsky-cameras. The setting up of such receiving equipment is economical and straightforward. Automatic uploading of fresh cloud imagery to the Web permits other users at an observatory site to share the data, as well as providing good public relations and educational outreach to interested persons.

\section{Acknowledgments}

We greatly appreciate advice from a number of people who helped with the establishment of our NOAA APT receiving station, particularly Craig Anderson (Abstract Technologies, Auckland, New Zealand) whose WXToImG software automates most of the operation. During
2005 June, Balthasar Indermuehle (UNSW) constructed an automated HRPT station in Sydney, and provided us with the HRPT images used within this paper.

\section{References}

Akerlof, C. E., et al. 2003, PASP, 115, 132

Anderson, C. T. 2005, www . wxtoimg . com

Davidoff, M. R. 1990, Radio Amateur's Satellite Handbook (Newington CT: American Radio Relay League)

Indermuehle, B. T. 2005, www . ozabove. com

Phillips, A. 2005, www.phys.unsw.edu.au/ map/ weather/notes/buildyourown/

Ryan, M. J. A. 2005, www. bcr.randwick. syd. catholic. edu.au/home/space/

Shamir, L., \& Nemiroff, R. J. 2005, PASP, in press

Straw, R. D. (ed.) 2003, ARRL Antenna Handbook 20th edn (Newington CT: American Radio Relay League) 